\title{
WEAK LENSING BY THE CLUSTER MS0302+1658
}

\author{
DAVID FISHER, KONRAD KUIJKEN AND MARIJN FRANX \\ Kapteyn Institute \\ PO Box 800, 9700 AV, Groningen, The Netherlands
}

\begin{abstract}
We present preliminary results of a weak lensing investigation of the $z=0.43$ cluster MS0302+1658.
\end{abstract}

We have started a program in Groningen to investigate the mass distributions in clusters of galaxies using weak gravitational lensing of distant background galaxies. Here we report preliminary results for the Einstein cluster MS0302+1658 (redshift 0.43), based on data obtained with the Wiliam Hershell Telescope. Total exposure times for the images analyzed were 7000 s in $\mathrm{R}$, and 6000 s in $\mathrm{V}$, and seeing was about 1 ".

This cluster contains two large central galaxies, with a 'straight arc' (Mathez et al. 1992) in between them, and a second arc in the envelope of the brightest cluster galaxy (Fig. 1a). Mathez et al. (1992) found the cluster potential to be poorly constrained by the arcs, since the central galaxies are a strong influence on them. We have therefore measured the weak lensing of background galaxies at larger radii (out to 2') by the cluster in order to provide a stronger measurement. At these radii the gravitational potential is dominated by the cluster as a whole, rather than by individual galaxies.

The data were analyzed following the methods of Kaiser, Squires and Broadhurst (1995). The V-R color-magnitude diagram indicates that most of the faint resolved objects are indeed blue galaxies, and probably lie behind the cluster. Averaging the complex second moments of the faint resolved objects and applying a (rather large and still preliminary) correction for circularization by seeing results in a 'polarization map': it gives the distortion of an intrinsically circular object at the mean source distance. Significant tangential distortion consistent with weak lensing is detected.

Comparison of these polarizations with a singular isothermal model results in a best-fit velocity dispersion of $700 \mathrm{~km} \mathrm{~s}^{-1}$, assuming the sources are at infinite distance and are all being lensed (Fig. 1b). Given the high cluster 

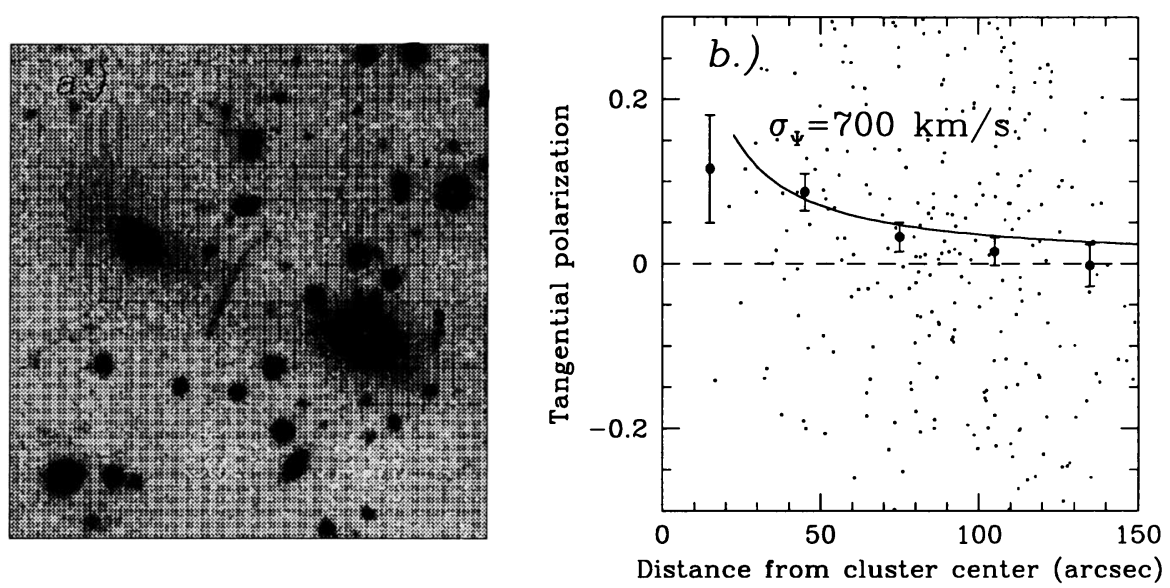

Figure 1. a.) The central $1^{\prime}$ of the cluster, containing the two large galaxies and giant arcs. b.) Elongations (small dots) of background galaxies and binned mean tangential polarizations (error bars) observed as a function of distance from the cluster center. The line shows the expected polarization of infinite-distance background sources by a $700 \mathrm{~km} \mathrm{~s}^{-1}$ singular isothermal sphere.

redshift, most sources are likely to be at most a factor of two further away than the cluster, reducing the efficiency of lensing. Therefore, the true velocity dispersion of the cluster potential is likely a factor 1.5-2 higher than the number quoted above. If the cluster potential is isothermal, we estimate a velocity dispersion of $\sigma_{\Psi}=1200 \mathrm{~km} \mathrm{~s}^{-1}$, but more careful study of the corrections for the seeing and finite source distances is necessary.

The galaxies in the cluster have a radial velocity dispersion of $920 \mathrm{~km} \mathrm{~s}^{-1}$ (Fabricant et al. 1994), and a deprojected radial number density $n_{g} \propto r^{-2.5}$. Thus, this cluster may provide an illustration of velocity bias: the galaxies are more centrally concentrated than the dark matter, and therefore show a smaller velocity dispersion. In a simple constant-velocity dispersion spherical cluster model with a power-law density and a singular isothermal potential, $n_{g}$ varies with radius as a power $-2\left(\sigma_{\Psi}^{2} / \sigma_{r}^{2}+\beta\right)$, where $\beta=1-\sigma_{t}^{2} / \sigma_{r}^{2}$ is the galaxy orbits' radial anisotropy. The line-of-sight velocity dispersion in such a model is $\sigma_{R V}^{2}=(1-12 \beta / 5) \sigma_{r}^{2}$, so the observed velocity dispersion and number count slope may imply a slight radial anisotropy $(\beta=0.15)$ of the galaxy orbits. A detailed error analysis as well as results for other cluster data in hand will be forthcoming.

\section{References}

Mathez, G., Fort, B., Mellier, Y., Picat, J.-P. \& Soucail, G. 1992, A\&A, 256, 343

Kaiser, N., Squires, G. \& Broadhurst, T. 1995, ApJ, 449, 460

Fabricant, D.G., Bautz, M.W. \& McClintock, J.E. 1994, AJ, 107, 8 